\title{
Estudio comparativo del lavado de manos convencional versus uso de solución hidroalcohólica durante la realización del intercambio peritoneal
}

\author{
María del Mar Baeza Valero, Antonia Gil Gil, Elena Gisbert Rosique \\ Enfermeras Servicio Nefrología. Hospital Universitario de Torrevieja. Alicante. España
}

\section{Sr. Director:}

Como se ha demostrado a lo largo de los años, la higiene de manos es un método básico para evitar la trasmisión de infecciones en la realización de los procesos sanitarios. El lavado de manos es una técnica que puede presentar diferentes estrategias en el momento de su aplicación y en especial en los procesos de diálisis peritoneal, donde un correcto lavado de manos será decisivo para el éxito de la técnica ${ }^{1}$.

Se evidencian dos técnicas básicas para el lavado de mano, una tradicional con agua y jabón y otra más reciente que utiliza las soluciones hidroalcohólicas. Estas últimas ofrecen ventajas frente al agua y jabón, obteniendo similares resultados de desinfección en un tiempo más reducido ${ }^{2}$, lo que las convierte en un método adecuado a seguir en los procesos del intercambio en diálisis peritoneal.

En el momento de la apertura de nuestra unidad seguíamos un protocolo que incluía un primer lavado de manos antes de la entrada del paciente a la habitación, donde se realizaría el intercambio peritoneal, y un segundo lavado de manos antes de la manipulación de la bolsa, ambos con agua y jabón. Posteriormente realizaban una desinfección de las manos, con solución hidroalcohólica, antes de la conexión y desconexión del catéter a la bolsa de líquido peritoneal.

Tras varios años siguiendo el método tradicional de desinfección de manos con agua y jabón, decidimos te-

\section{Correspondencia:}

$M^{\text {a }}$ del Mar Baeza Valero

Hospital U. de Torrevieja. Servicio de Nefrología

Carretera CV 95 s/n. 03186 Torrevieja. Alicante

E-mail:mmbaeza@torrevieja-salud.com ner en cuenta las nuevas evidencias científicas sobre la desinfección de manos ${ }^{2-4}$ y acordamos sustituir el segundo lavado de manos previo a la manipulación de las bolsas de diálisis con agua y jabón por el uso de los desinfectantes hidroalcohólicos.

La finalidad de esta modificación sería, por tanto, proporcionar al paciente mayor seguridad y calidad, realizando el procedimiento correcto y reduciendo el tiempo de realización del intercambio peritoneal al evitar un segundo desplazamiento al baño tras preparar el material.

Nos planteamos como objetivo comparar la eficacia de dos procedimientos para la higiene de las manos durante la realización del intercambio peritoneal. El primer procedimiento con un lavado de manos con agua y jabón previo a la manipulación de la bolsa de líquido peritoneal y el segundo con una desinfección con solución hidroalcohólica en ese mismo momento, analizando la tasa anual de peritonitis en cada uno de los procedimientos.

\section{Material y Método}

Realizamos un estudio observacional descriptivo de 4 años de duración, en un periodo comprendido desde enero de 2014 hasta diciembre de 2017. Dividimos este periodo de tiempo en dos intervalos de dos años cada uno.

En el estudio se incluye a todos los pacientes que iniciaron diálisis peritoneal durante los periodos analizados, siendo un total de 40 pacientes (no se aplican criterios de exclusión).

Los pacientes se realizaban la diálisis peritoneal ellos mismos a excepción de un paciente, incluido en el segundo periodo, al que varios cuidadores le realizaban el intercambio. 
Periodo 1. En el intervalo correspondiente a los 2 primeros años (2014-2015) se sigue el procedimiento de realización del intercambio peritoneal que incluye un lavado de manos previo a la preparación del material y un segundo lavado previo a la manipulación de la bolsa ambos con aguja y jabón. En el total del primer periodo la unidad contó con 21 nuevos pacientes que fueron entrenados con este procedimiento.

Subrayar, que estos pacientes siguieron con el procedimiento en el que fueron formados (dos lavados de manos con agua y jabón) hasta el abandono de la técnica, por lo que no se contabilizan dentro del segundo periodo ya que no se les formó con el nuevo procedimiento.

Periodo 2. En el segundo intervalo que corresponde a los 2 años siguientes (2016-2017) se sustituye el segundo lavado de manos con agua y jabón por una desinfección con una solución hidroalcohólica al 75 \%. En este segundo periodo son incluidos los pacientes nuevos que comenzaron diálisis y fueron entrenados con este procedimiento siendo un total de 19.

Se definió la peritonitis ${ }^{5}$ como la presencia de al menos dos de los siguientes criterios:

- Síntomas como dolor abdominal, vómitos diarrea, sensación de fiebre y escalofríos o febrícula, y signos de inflamación peritoneal, rebote peritoneal.

- Presencia de líquido peritoneal turbio con un aumento de la celularidad, con más de 100 leucocitos/ $\mu \mathrm{l}$ en el recuento celular, y más del $50 \%$ polimorfonucleares en la fórmula.

- Presencia de microorganismos en el efluente peritoneal mediante tinción Gran o cultivo del líquido peritoneal.

Se recogieron todos los datos en una hoja de Excel y se calculó la tasa anual de peritonitis por año en ambos periodos (número de peritonitis en el periodo de estudio / [número de pacientes del periodo de estudio $x$ número de años del periodo de estudio]), teniendo en cuenta el tiempo desde que el paciente inicia la diálisis peritoneal en el domicilio, hasta la baja definitiva en la técnica, descartando el tiempo de entrenamiento. No se tuvieron en cuenta las peritonitis catéter dependiente.

\section{Resultados}

De los 21 pacientes estudiados en el primer periodo, la media de edad fue de 65 años, 3 eran mujeres y 18 hombres, siendo 8 de los individuos de nacionalidad extranjera.

En el segundo periodo se estudiaron 19 pacientes, cuya media de edad fue de 58 años, 7 de ellos eran mujeres y 12 hombres, con una población extranjera de 7 pacientes.

En el primer periodo se obtiene un ratio de 0,17 peritonitis/pacientes y año, mientras que en el segundo periodo el ratio fue de 0,18 peritonitis/pacientes y año.

\section{Discusión y Conclusiones}

Observamos que en ambos procedimientos el ratio de peritonitis entra dentro de los estándares de calidad que se indican en plan de calidad científico técnico y de mejora continua de calidad en diálisis peritoneal' ${ }^{6}$.

Aunque no hemos encontrado diferencias significativas entre ambos procedimientos en los periodos estudiados, al evitar un segundo desplazamiento al baño, se beneficiaron de un acortamiento en el tiempo destinado a la realización del intercambio.

Además es de destacar, que al eliminar el recorrido al baño para realizar un lavado de mano tradicional y sustituirlo por un lavado por fricción, se reducen los riesgos potenciales inherentes al recorrido, tales como caídas accidentales, manipulación incorrecta de zonas no asépticas etc. Además consideramos que este nuevo procedimiento, podría ofrecer a nuestros pacientes mayor confort, seguridad y rapidez.

Es preciso resaltar que debido a que la muestra a estudio es muy pequeña, nuestros resultados no son concluyentes. Sería recomendable nuevas líneas de investigación en esta vía con la finalidad de mejorar la calidad y seguridad del paciente.

En nuestra Unidad a día de hoy seguimos el procedimiento que incluye la desinfección hidroalcohólica, debido a los buenos resultados obtenidos de seguridad, calidad y optimización del tiempo en la realización del procedimiento.

En base a los resultados obtenidos consideramos que el lavado de manos con agua y jabón junto a la utilización 
de solución hidroalcohólica ofrece resultados adecuados en la técnica de diálisis peritoneal.

Recibido: 28 noviembre 2018

Revisado: 14 diciembre 2018

Modificado: 27 diciembre 2018

Aceptado: 10 enero 2019

\section{Bibliografía}

1. Granado A, Ruiz C, Arrieta J. Adiestramiento del paciente que se incorpora a diálisis peritoneal. Visitas domiciliarias. Reentrenamiento. En: Coronel $F$, Molina A, Caravaca F, editores. Guías de Práctica Clínica en Diálisis Peritoneal. Madrid: Sociedad Española de Nefrología (SEN);2005. p.114-5.

2. Allegranzi B, Bagheri Nejad S, Chraiti M. Aplicación de la estrategia multimodal para la mejora de la higiene de las manos y el conjunto de herramientas en la asistencia ambulatoria. En: Rosemary Sudan, editor. La higiene de las manos en la asistencia ambulatoria y domiciliaria y en los cuidados de larga duración: guía de aplicación de la estrategia multimodal de la OMS para la mejora de la higiene de las manos y del modelo Los cinco momentos para la higiene de las manos. Ginebra: Organización Mundial de la Salud (OMS);2013. p.18.

3. Estrategia de Seguridad del Paciente del Sistema Nacional de Salud 2015-202. Ministerio de Sanidad y Bienestar Social Gobierno de España. Madrid. [Acceso 15 Ene 2018]. Disponible en: https:// www.seguridaddelpaciente.es/

4. Pittet D, Hugonnet $S$, Harbarth S, Mourouga $P$, Sauvan $V$, Touveneau $S$ et al. Effectiveness of a hospital-wide programme to improve compliance with hand hygiene. Infection Control Programme. Lancet. 2000;356(9238):1307-12.

5. Montenegro M. Peritonitis e infecciones del catéter en la diálisis peritoneal. Nefrología al día Sociedad Española de Nefrología. [Internet] 2018 [Consultado 1 0ct 2018]. [aprox 12 p]. Disponible en: http://www.nefrologiaaldia.org/es-articulo-peritonitis-e-infecciones-del-cateter-53

6. Álvarez-Ude F, Arenas D, Arrieta F, Bajo M, Borras $\mathrm{M}$, Coronel $\mathrm{F}$ et al. Plan de calidad científico técnica y de mejora continua de calidad en diálisis peritoneal (DP). Madrid: Sociedad Española de Nefrología (SEN);2017. p. 16-7.

Este artículo se distribuye bajo una Licencia Creative Commons Atribución-NoComercial 4.0 Internacional. https://creativecommons.org/licenses/by-nc/4.0/ 